\title{
EL GÉNERO TRIEPEOLUS ROBERTSON, 1901 EN LA HISPANIOLA, CON LA DESCRIPCIÓN DE UNA ESPECIE NUEVA (HYMENOPTERA: ANTHOPHILA: APIDAE)
}

\author{
Julio A. Genaro \\ Investigador Asociado del Museo Nacional de Historia Natural "Prof. Eugenio de Jesús Marcano". \\ Calle César Nicolás Penson, Plaza de la Cultura, Santo Domingo, República Dominicana. \\ polimita@hotmail.com
}

\section{RESUMEN}

Se revisan las abejas cleptoparasíticas del género Triepeolus que habitan en La Hispaniola. Una especie nueva: $T$. danieli y la hembra previamente desconocida de $T$. nisibonensis se describen. Con esta descripción el número de especies se eleva a tres. Se presenta la distribución en la isla, amplitud geográfica, época de vuelo y una clave para separar las especies.

Palabras clave: abejas parasíticas, Apoidea, Apidae, Nomadinae, Epeolini, Triepeolus, especie nueva, revisión taxonómica, Hispaniola.

Title: The genus Triepeolus Robertson, 1901 in Hispaniola, with the description of a new species (Hymenoptera: Anthophila: Apidae).

\section{ABSTRACT}

The Hispaniolan cleptoparasitic bees of the genus Triepeolus are revised. One new species: T. danieli and the female of T. nisibonensis are described. With this new taxon the number of species is elevated to three. Distribution, geographical range, flight season, and a key to the species are presented.

Keywords: Cuckoo bee, Apoidea, Apidae, Nomadinae, Epeolini, Triepeolus, new species, taxonomic revision, Hispaniola.

\section{INTRODUCCIÓN}

Las abejas del género Triepeolus se caracterizan principalmente por tener dos o tres segmentos palpales; dos bandas longitudinales de pubescencia clara, corta y ajustada, que se extienden desde el margen anterior hasta casi la mitad del escudo y esterno 7 (S7) del macho, con pelos desarrollados en la emarginación apicolateral del margen posterior (Rightmyer, 2004; Michener, 2007). Estas abejas son parasíticas y superficialmente parecen avispas por los colores blanco-amarillento, amarillo o naranja y negro, además de tener pubescencia escasa.

Las especies antillanas de Triepeolus, hasta el momento, se conocen de Cuba: T. cuabitensis Genaro, T. roni Genaro, T. vicinus (Cresson), T. wilsoni (Cresson); Las Bahamas: T. roni y T. vicinus; Hispaniola: T. nisibonensis Genaro y T. victori Genaro; Jamaica, Granada, México, EE.UU: T. rufoclypeus (Fox) (=T. foxii Cockerell); Granada, San Vicente y Las Granadinas: T. rufotegularis (Ashmead). Moure y Melo (2007) listan las especies Neotropicales, aunque mencionan erróneamente a Haití y México dentro de la distribución de T. wilsoni.

El género fue revisado en Cuba por Genaro (1999), adicionando dos especies nuevas; el mismo autor describió dos especies nuevas para La Hispaniola (Genaro, 1998, 2001). 
Rightmyer (2004; 2008) revisó la tribu Epeolini, presentó claves y describió especies nuevas del Nuevo Mundo.

\section{OBJETIVOS}

- Describir una especie nueva del género Triepeolus para La Hispaniola.

- Describir la hembra de T. nisibonensis.

- Presentar una clave para la separación de las especies del género.

\section{MATERIALES Y MÉTODOS}

Los términos morfológicos utilizados siguen lo propuesto por Rightmyer (2008). Las abreviaturas son usadas como sigue: cada tergo o esterno metasomal es llamado $\mathrm{T}$ o $\mathrm{S}$, respectivamente, seguido por un número que lo define específicamente, ejemplo: T1 es el primer tergo metasomal y $\mathrm{S} 1$ el primer esterno. La densidad de las puntuaciones está dada en términos de la relación entre el diámetro de la puntuación (d) y el espacio (e) entre ellas, como ejemplo $\mathrm{e}=2 \mathrm{~d}$, para demostrar su separación.

El material estudiado está depositado en las siguientes colecciones: Canadá: colección del autor (JAG), Estados Unidos: National Museum of Natural History, Institución Smithsoniana (NMNH), Washington, D.C.; República Dominicana: Museo Nacional de Historia Natural "Profesor Eugenio de Jesús Marcano" (MNHNSD).

\section{SISTEMÁTICA}

Clave para separar las especies de Triepeolus de La Hipaniola

Machos con 13 segmentos antenales; siete tergos metasomales expuestos y placa pigidial en T7 (Fig. 1). Hembras con 12 segmentos antenales; seis tergos metasomales expuestos y área seudopigidial consistente de setas especializadas, ubicadas en la región medioapical de T5 (Fig. 2).

1. Especie robusta, más de $14 \mathrm{~mm}$ de longitud corporal (Fig. 3). Pelos simples, largos, erectos en episterno. .victori

Especies no robustas, menos de 14 mm de longitud corporal (Figs. 4 y 5). Pelos plumosos, cortos, recostados en mesepisterno. 2

2. Tamaño mediano (alrededor de 8-12 mm de longitud corporal). Margen dorsal de la cabeza escotado (Fig. 6). Escutelo bi-giboso (Fig. 7). nisibonensis

Tamaño pequeño (alrededor de 6-7 mm de longitud corporal). Margen dorsal de la cabeza no escotado (Fig. 8). Escutelo plano, sin gibas (Fig. 8). danieli sp. nov.

Orden Hymenoptera: Superfamilia Apoidea, Familia Apidae, Subfamilia Nomadinae, Tribu Epeolini Triepeolus danieli sp. nov.

Diagnosis. Tamaño pequeño. Tegumento negro, excepto áreas como las patas, lóbulo pronotal, tégula, escutelo y axila castaño rojizo. Bandas apicales transversas de pubescencia plumosa 

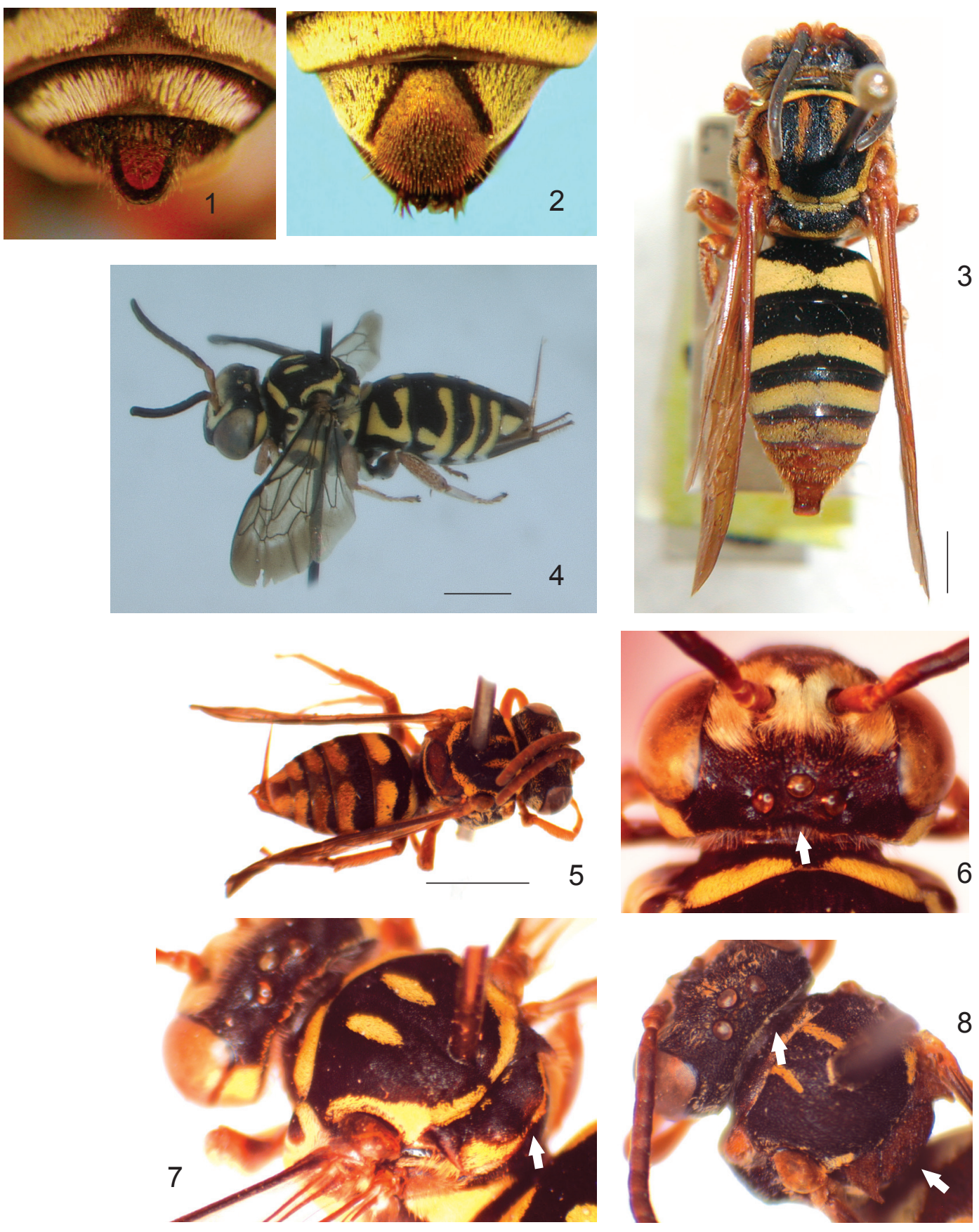

Figuras 1-8. Especies de Triepeolus. 1. Placa pigidial de un macho en T7. 2. Área seudopigidial de una hembra en T5. 3. Hábito de T. victori macho. 4. Hábito de T. nisibonensis hembra. 5. Hábito de T. danieli sp. nov. 6. Margen dorsal de la cabeza escotado, en T. nisibonensis hembra. 7. Escutelo bi-giboso, en T. nisibonensis hembra. 8. Margen dorsal de la cabeza no escotado y escutelo plano, no bigiboso, en $T$. danieli sp. nov. hembra. Escala de las Figs. 3, 4 y $5=2 \mathrm{~mm}$. 
amarilla interrumpidas en el centro. Área seudoapical poco diferenciada del resto de T5, semitriangular con setas amarillo claro brillantes.

Diagnosis. Small size. Body black except some areas like legs, pronotal lobe, tegula, scutellum and axilla, reddish brown. Yellow apical transverse bands of terga interrupted in the middle. Pseudopygidial area vaguely distinct from rest of T5, semi-triangular with shining pale yellow setae.

Diagnosis diferencial. T. danieli sp. nov. se diferencia por su menor talla del resto de las especies de La Hispaniola. Solamente pudiera ser confundida con T. cuabitensis, de la cual se separa fundamentalmente por el patrón metasomal de las bandas apicales transversas (entera en T4 de T. cuabitensis); la puntuación del mesepisterno (grandes y unidas en T. cuabitensis); escutelo castaño rojizo y plano (en T. cuabitensis es negro y bigiboso); area seudopigidial (más definida, alargada y con mayor desarrollo de las setas en T. cuabitensis).

Descripción. Hembra. Longitud corporal total. 6,0-6,4 mm. Longitud ala anterior: 5,0-5,2 mm.

Coloración. Tegumento negro con las siguientes áreas castaño rojizo: labro; escapo antenal; antenas, sobre todo ventralmente; coxas; patas; lóbulos pronotales; escutelo; axilas y S6. Alas castaño, más oscurecidas en ápice; nervaduras castaño oscuro.

Pubescencia. Densa, corta, plumosa, amarilla: en labro; clípeo; área supraclipeal; gena; banda transversa sobre pronoto; dos bandas longitudinales paralelas que se extienden hacia centro del escudo; márgenes de lóbulos pronotales; banda que bordea el escudo, excepto margen anterior; bordeando mesepisterno, metepisterno, superficie posterior del propodeo; formando bandas transversas apicales interrumpidas en el centro en T1-T5; banda transversa basal en T1; pubescencia más clara en región ventral. Placa seudopigidial con setas amarillo, más claro y brillante. Pubescencia negra en áreas de tegumento negro en metasoma.

Estructura. Superficie del escutelo plana, sin dos gibas características de otras especies. Axilas triangulares, con la base ancha y algo alargadas, sobrepasando el escutelo. Área seudopigidial apenas diferenciada del resto de T5, más ancha que larga, semi-triangular.

Esculturación. Cabeza. Labro, clípeo, área supraclipeal, frente, vértex punteado-reticulado; gena con puntuaciones similares pero algo más reticulado. Mesosoma. Escudo con puntuaciones como en la cabeza; tégula con puntuaciones muy pequeñas, irregulares $(\mathrm{e}=0,5-1,5 \mathrm{~d})$; axila, escutelo puntuado-reticulado; área central del escutelo con puntuaciones mayores; mesepisterno irregularmente punteado ( $\mathrm{e}=0-1 \mathrm{~d})$, puntuaciones alargadas, puntuado-reticulado en área dorsolateral; área hipoepimeral puntuada-reticulada con puntuaciones mayores en centro; metepisterno irregularmente punteado $(\mathrm{e}=0,5-2 \mathrm{~d})$; puntuaciones del propodeo como en escudo, pero de menor tamaño. Metasoma. Tergos y esternos con puntuaciones más pequeñas que mesosoma, unidas $(\mathrm{e}=0)$.

Macho desconocido.

Tipos. Holotipo hembra. HISPANIOLA: República Dominicana, Cueva del Puente, Guaraguao, Parque Nacional del Este, 9.v.1992, col. B. Hierro (MNHNSD). Paratipo hembra, con los mismos datos, depositada en NMNH.

Etimología. Dedicada con especial cariño a mi hijo Daniel E. Genaro. 


\section{Triepeolus nisibonensis Genaro.}

Triepeolus nisibonensis Genaro 2001. Rev. Biol. Trop. 49: 1033. Holotipo macho, FSCA.

Diagnosis. Patrón de bandas apicales transversas de pubescencia amarilla, interrumpidas en el centro (Fig. 4), algunos machos en lugar de amarillo pueden tener la pubescencia blanca; margen dorsal de la cabeza escotado (Fig. 6). Mesepisterno con pelos plumosos, recostados; escutelo bi-giboso (Fig. 7).

Descripción. Hembra. Longitud corporal total. 10,0-10,4 mm. Longitud ala superior: 7,9-8,2 $\mathrm{mm}$.

Similar al macho, excepto: pubescencia corta recostada de las bandas, amarillo en los ejemplares examinados. Carece de pelos largos modificados en centro del margen posterior de S3-S5. Área seudopigidial triangular, castaño rojizo, con setas castaño rojizo cortas, cubriendo algo más de la mitad; las más alargadas, en región basal. Procesos largos de S6, con varias cerdas a todo lo largo, terminado en espinas curvadas.

Material examinado. HISPANIOLA, República Dominicana, Cibao Oriental, Loma Quita Espuela, Centro Visitantes, ii.2002, col. J. A. Genaro (3 hembras, NMNH, JAG).

Meses de actividad. Febrero, mayo.

Triepeolus victori Genaro.

Triepeolus victori Genaro 1998. Caribbean J. Sc., 34: 92. Holotipo hembra, FSCA.

Diagnosis. Especie robusta. Patrón de bandas apicales transversas de pubescencia amarilla casi o completamente continuas (Fig. 3); mesepisterno con pelos erectos, simples; escutelo bi-giboso.

Meses de actividad. Junio, julio.

\section{CONCLUSIÓN}

Hasta el momento todas las especies de este género son endémicas de La Hispaniola. La similitud faunística entre La Hispaniola y Cuba o La Hispaniola y Puerto Rico es conocida y apoyada por ejemplos de especies parecidas (Genaro, 2007; 2009), muy emparentadas, que habitan en cada isla, como producto de una historia paleogeográfica común (Iturralde-Vinent y MacPhee, 1999). En este sentido, el género Triepeolus presenta cuatro especies hermanas, posiblemente vicariantes, que fundamentan los mecanismos de poblamiento a partir de ancestros comunes, aislamiento y especiación: T. victori-T. wilsoni y T. cuabitensis-T. danieli sp. nov .

\section{AGRADECIMIENTOS}

A los administradores de colecciones del MNHNSD: Kelvin Guerrero, en el momento del préstamo y Carlos Suriel, actualmente, por las facilidades brindadas durante el estudio de las colecciones y la facilitación para el préstamo de material. A Carlos Suriel por permitirme la deposición del paratipo en NMNH. A los miembros de la Fundación Loma Quita Espuela, San Francisco de Macorís, por el apoyo ofrecido durante el trabajo de campo en la zona. A Antonia Guidotti (Royal Ontario Museum, Toronto) por asentir el acceso al equipamiento y facilitar la toma de algunas fotografías. A Esteban Gutiérrez, un revisor anónimo, así como al editor, por sus acertadas sugerencias durante la lectura del manuscrito original. 


\section{LITERATURA CITADA}

Genaro, J. A. 1998. Especie nueva de Triepeolus para República Dominicana (Hymenoptera: Anthophoridae). Caribbean Journal of Science, 34: 92-94.

Genaro, J. A. 1999. Revisión del género Triepeolus en Cuba (Hymenoptera: Apidae), con descripción de dos especies nuevas. Caribbean Journal of Science, 35: 215-220.

Genaro, J. A. 2001. Especies nuevas de abejas de Cuba y la Española (Hymenoptera: Colletidae, Megachilidae, Apidae). Revista de Biología Tropical, 49: 951-959.

Genaro, J. A. 2007. Especie nueva de Zanysson y primer registro de Epinysson borinquinensis para Hispaniola (Hymenoptera: Apoidea, Crabronidae). Solenodon, 6: 52-59.

Genaro, J. A. 2009. Dos especies nuevas de Cerceris de las Antillas Mayores (Hymenoptera: Apoidea, Crabronidae), con notas sobre su historia natural. Solenodon, 8: 99-117.

Iturralde-Vinent, M. y R. MacPhee. 1999. Paleogeography of the Caribbean region, implications for Cenozoic biogeography. Bulletin of the American Museum of Natural History, 238: $1-95$.

Michener C. D. 2007. The bees of the world, second edition. Johns Hopkins University Press, Baltimore. 953 pp.

Moure, J. S. y G. A. R. Melo. 2007. Nomadini Latreille, 1802. Pp: 578-599. In: J. S. Moure; D. Urban y G. A. R. Melo. Catalogue of Bees (Hymenoptera, Apoidea) in the Neotropical Region. Sociedade Brasileira de Entomologia, Curitiba.

Rightmyer, M. G. 2004. Phylogeny and classification of the parasitic bee tribe Epeolini (Hymenoptera: Apidae, Nomadinae). Scientific Papers. Natural History Museum, The University of Kansas, 33:1-51.

Rightmyer, M. G. 2008. A review of the cleptoparasitic bee genus Triepeolus (Hymenoptera: Apidae). Part I. Zootaxa, 1710: 1-170.

[Recibido: 15 de julio, 2014. Aceptado para publicación: 27 de agosto, 2014] 